\title{
Excitation spectrum and electrical properties of the condensate of Bose atoms
}

\author{
A.M.Kosevich \\ B.Verkin Institute for Low Temperature Physics and Engineering \\ of National Academy of Sciences of Ukraine, \\ 47, Lenin Avenue, Kharkiv, 61103 Ukraine
}

Received July 15, 2005, in final form October 24, 2005

It is shown that the condensate of a degenerated Bose gas consisting of neutral atoms possesses electrical properties which differ from a trivial polarization of the atoms in the electric field. A notion of an isotropic quadrupole moment (IQM) of a neutral atom is introduced. A distribution of IQM reflects a specific spatial ordering in the condensate and produces a distribution of the electric potential. Small vibrations of the Bose gas are considered and a correction to the Bogoliubov spectrum of elementary excitations in the degenerated Bose gas is obtained. An additional term in the Gross-Pitaevskii equation which is responsible for such a correction is found and a new type of the nonlinear Schrödinger equation (NSE) is constructed. Since the Bose condensate is akin to the superfluid component in $\mathrm{He} \mathrm{II}$, a manifestation of its electrical activity could have a relation to the electrical activity of the superfluid liquid observed experimentally.

Key words: Bose condensate, Bogoliubov spectrum, electrical polarization of $\mathrm{He}$ II

PACS: 47.27.Eq, 67.40.Db

\section{Electrical activity of neutral atoms}

The neutral He atom in the $1 S_{0}$ ground state does not have an intrinsic ( in the absence of electrical field ) dipole moment but it does have an important microscopic electrical characteristic [1] - an isotropic quadrupole moment (IQM). The IQM is defined by the following formula

$$
q_{i k}=\sum \mathrm{e} x_{i} x_{k}, \quad i, k=1,2,3,
$$

where the summation is over all electric charges in the system. In the case of the $\mathrm{He}^{4}$ we have $q_{i k}=q_{0} \delta_{i k}$ and $q_{0}=(1 / 3) q_{l l}=-2|\mathrm{e}| a^{2}$ (e is the charge of the electron and $a$ is the Bohr radius). From the standpoint of macroscopic physics this is a 
"latent" atomic characteristic, since in macroscopic interactions of electrical systems the quantity $q_{i k}-(1 / 3) q_{0} \delta_{i k}$ goes to zero in the given case.

The IQM produces an electric potential inside the atom. To calculate the potential we use the simplest classical model of an atom, supposing all the electrons distributed homogeneously over the surface of a sphere with radius $a$. If the total charge of the atomic nucleus is $Z|\mathrm{e}|$, the electrostatic potential averaged by volume inside such a sphere-atom having the volume $V_{0}=4 \pi a^{3} / 3$ is equal to $\varphi_{0}=3 Z|\mathrm{e}| /((2 a)$. According to the model proposed, the atomic IQM is equal to $q_{0}=-Z|\mathrm{e}| a^{2}$.

Since the potential is concentrated inside the atoms, its spatial localized distribution in a gas may be described in the long wave approximation by means of the expression

$$
\varphi(\mathbf{x})=\varphi_{0} V_{0} \sum_{\alpha} \delta\left(\mathbf{x}-\mathbf{x}_{\alpha}\right)=-2 \pi q_{0} \sum_{\alpha} \delta\left(\mathbf{x}-\mathbf{x}_{\alpha}\right)
$$

where $\mathbf{x}_{\alpha}$ is a coordinate of $\alpha$-atom and the summation is extended over all the atoms in the system. The long wave formula (1) was derived strongly in the paper [2].

Let us introduce a microscopic density of atoms

$$
n(\mathbf{x})=\sum_{\alpha} \delta\left(\mathbf{x}-\mathbf{x}_{\alpha}\right)
$$

As a result, we obtain a connection of the electric potential of a neutral gas $\varphi(\mathbf{x})$ with the density of the atomic IQMs $Q$ :

$$
\varphi(\mathbf{x})=-2 \pi Q, \quad Q=q_{0} n(\mathbf{x}) .
$$

Equations (2), (3) make it possible to calculate the mean electric potential in the condensate. Consider the Bose condensate either in the ground state or in a slightly excited state. The atoms in the coherent condensate state form some ordered structure with the wave function

$$
\Psi\left(\mathbf{x}_{\alpha}\right)=\Psi\left(\mathbf{x}_{1}, \mathbf{x}_{2}, \mathbf{x}_{3}, \ldots\right) .
$$

In order to describe the slightly excited states of a nearly ideal Bose gas we suggest to use the approximation of a mean field type. Suppose all the atoms are found in the same single-particle quantum state $\psi_{0}\left(\mathbf{x}_{\alpha}\right)$ :

$$
\Psi\left(\mathbf{x}_{\alpha}\right)=\prod_{\alpha} \psi_{0}\left(\mathbf{x}_{\alpha}\right)
$$

Then the mean density of the gas has got a very simple form

$$
\begin{aligned}
\langle n(\mathbf{x})\rangle & =\sum_{\alpha}\left\langle\Psi\left|\delta\left(\mathbf{x}-\mathbf{x}_{\alpha}\right)\right| \Psi\right\rangle=\sum_{\alpha} \int|\Psi|^{2} \delta\left(\mathbf{x}-\mathbf{x}_{\alpha}\right) \prod_{\beta} \mathrm{d} V_{\beta} \\
& =\sum_{\alpha}\left|\psi_{0}(\mathbf{x})\right|^{2}=N_{0}\left|\psi_{0}(\mathbf{x})\right|^{2}
\end{aligned}
$$

where $N_{0}$ is the number of atoms in the condensate. Of course the function $\psi_{0}(\mathbf{x})$ is normalized to unity. 
The problem of describing the electrical properties of the condensate comes to a problem of searching the function $\psi_{0}(\mathbf{x})$. However in the mean field approximation, the function $\psi_{0}(\mathbf{x})$ can be taken in the form of a solution of the Gross-Pitaevskii equation [3]:

$$
\mathrm{i} \hbar \frac{\partial \psi_{0}}{\partial t}=-\frac{\hbar^{2}}{2 m} \Delta \psi_{0}+U_{0}\left(\left|\psi_{0}\right|^{2}-n_{0}\right) \psi_{0}
$$

where $n_{0}=N_{0} / V$ is the equilibrium density of the condensate, and a solution of equation (6) should be normalized to the number of atoms $N_{0}$.

Slightly excited states of the condensate are equivalent to small vibrations and the wave function of such vibrations can be written as follows:

$$
\psi(\mathbf{x}, t)=\sqrt{n}_{0}(1+\theta(\mathbf{x}, t)), \quad|\theta(\mathbf{x}, t)| \ll 1 .
$$

Linearization of equation (6) with respect to $\theta(\mathbf{x}, t)$ leads to the following equation

$$
\mathrm{i} \hbar \frac{\partial \theta}{\partial t}=-\frac{\hbar^{2}}{2 m} \Delta \theta+U_{0} n_{0}\left(\theta+\theta^{*}\right) .
$$

Equation (8) comes to two partial differential equations with the time derivatives of the second order with respect to the $\operatorname{sum} \theta+\theta^{*}$ and the difference $\theta-\theta^{*}$. We take the solution

$$
\theta+\theta^{*}=\theta_{0} \cos (\mathbf{k} \mathbf{x}-\omega t)
$$

where $\omega$ and $\mathbf{k}$ are the frequency and wave vector connected by means of the formula for Bogoliubov's spectrum

$$
\omega^{2}=k^{2}\left(\frac{U_{0} n_{0}}{m}+\left(\frac{\hbar k}{2 m}\right)^{2}\right) .
$$

Equation (9) makes it possible to calculate the small oscillations if the gas density (6):

$$
\delta n=n_{0} \theta_{0} \cos (\mathbf{k} \mathbf{x}-\omega t) .
$$

The oscillations of the density produce oscillations of the electric potential (3) in the neutral Bose gas. This effect can have a relation to the oscillations of the electric potential produced by the vibrations of the second sound wave in a superfluid liquid observed by Rybalko [4]. Really, eigen solutions of equation (8) describe elementary excitations in the condensate and $\delta n$ can be treated as a density of elementary excitations above the ground state. In the theory of superfluidity [3] the vibrations of the density of elementary excitations are associated with temperature oscillations. In its turn a nondissipative wave of the temperature oscillations is an analog of the second sound wave in the helium II.

\section{Correction to Bogoliubov's spectrum}

The electrical activity of the Bose condensate should be taken into consideration in solving the problem of its small vibrations. We restrict ourselves to the mean field approximation and long wave Gross-Pitaevskii equation. 
In the long wave approximation, the interaction of an atom in the point $\mathbf{x}_{\mathbf{1}}$ with an external field $\varphi(\mathbf{x})$ is as follows:

$$
\delta U_{\mathrm{int}}=\frac{1}{2} q \Delta \varphi\left(\mathbf{x}_{\mathbf{1}}\right)
$$

where $q$ is the atomic IQM. If the field $\varphi(\mathbf{x})$ is created by an atom in the point $\mathbf{x}_{\mathbf{2}}$, we can use the expression (1) for the potential produced by the second atom. Then in the long wave approximation the energy of the pair interaction of the atoms under consideration can be written as

$$
U\left(\mathbf{x}_{1}-\mathbf{x}_{2}\right)=-2 \pi q^{2} \Delta \delta\left(\mathbf{x}_{1}-\mathbf{x}_{2}\right) .
$$

The Fourier component of this interaction energy is equal to

$$
U_{k}=\pi q^{2} k^{2}
$$

However, equation (13) for the pair interaction energy does not include all possible contact interactions of two atoms. In particular, it does not take into consideration two important facts. First of all, a finite size of the atomic radius $a$ should be taken into account [5]. And secondly, a deformation of the electron distributions inside the atoms during their contact was excluded from the calculation of equation (12). These facts give an additional contribution to the Fourier component (14) and can change both the magnitude and the sign of $U_{k}$. Consequently, the total pair energy of the atomic interaction in the long wave approximation should be written as follows:

$$
U_{k}=U_{0}+U_{1}(a k)^{2},
$$

where the parameter $U_{1}$ has an order of the magnitude of $U_{0}$.

A new form of the second term in equation (15) changes the interaction energy in the coordinate representation

$$
\delta U\left(\mathbf{x}_{1}-\mathbf{x}_{2}\right)=-U_{1} \Delta \delta\left(\mathbf{x}_{1}-\mathbf{x}_{2}\right)
$$

The additional term in equation (15) which is proportional to $k^{2}$ also leads to the change of Bogoliubov 's spectrum. Now small vibrations of the condensate have the following frequencies squared

$$
(\hbar \omega)^{2}=\varepsilon^{2}(p)+(\hbar \Omega(p))^{2},
$$

where $\varepsilon(p)$ is given by equation (10) and the frequency $\Omega$ plays the role of an ion plasma frequency:

$$
\Omega^{2}(p)=\frac{U_{1} n_{0}}{m}\left(\frac{p}{\hbar}\right)^{4} \equiv \frac{U_{1} n_{0}}{m} k^{4} .
$$

Taking into account the electrical activity of the Bose condensate, one does not change the low frequency spectrum of the sound vibrations. We see only a slight renormalization of the mass of elementary excitations at high frequencies. 
In its turn the expression (16) enables us to determine an additional term in the Gross-Pitaevskii equation associated with the electrical activity of the condensate. Considering (6) as an equation derived in the mean field approximation, we can include the term (16) averaged over the state $\psi_{0}$ into the effective Hamiltonian. Since

$$
\left\langle\Psi_{0}\left(\mathbf{x}_{1}\right)\left|\delta U\left(\mathbf{x}-\mathbf{x}_{1}\right)\right| \Psi_{0}\left(\mathbf{x}_{\mathbf{1}}\right)\right\rangle=-U_{1} \Delta\left|\Psi_{0}(\mathbf{x})\right|^{2},
$$

we proposed [6] a little bit changed form of equation (6):

$$
\mathrm{i} \hbar \frac{\partial \Psi_{0}}{\partial t}=-\frac{\hbar^{2}}{2 m} \Delta \Psi_{0}+U_{0}\left(\left|\Psi_{0}\right|^{2}-n_{0}\right) \Psi_{0}-U_{1} \Psi_{0} \Delta\left|\Psi_{0}\right|^{2} .
$$

Thus, taking the electrical properties of the Bose condensate into account we come to a new type of the nonlinear Schröeding equation (NSE) describing the dynamics of the Bose condensate.

\section{Remarks}

Return to equations (1)-(3):

$$
\varphi(\mathbf{x})=-2 \pi q n(\mathbf{x}), \quad n(\mathbf{x})=\sum_{\alpha} \delta\left(\mathbf{x}-\mathbf{x}_{\alpha}\right) .
$$

This expression has a relation for any gas and for any condensed matter.

Using statistical-thermodynamical averaging of equation (21) one can write

$$
\langle\langle\varphi\rangle\rangle=-2 \pi\langle\langle q n\rangle\rangle
$$

In the case of a gas the parameter $q$ is a characteristic of a single atom and does not depend on the states of other atoms of the gas: $\langle\langle q n\rangle\rangle=q\langle\langle n\rangle\rangle$. In the case of a condensed matter, a distribution of the electron charge in any atom depends on the neighbouring atoms and their states, and a calculation of the parameter $q$ is a special problem of the quantum mechanics of many particles. As a result the mean electric potential (22) depends not only on the atom radius $a$ but on the mean interatomic distance in the matter $l$ as well. 


\title{
References
}

1. Kosevich A.M., On the description of electrical effects in the two-fluid model of superfluidity. FNT, 2005, 31, No. 1, 50-54 (in Russian); Low Temp. Phys, 2005, 31, No. 1, $37-40$.

2. Kosevich A.M., Dynamic electrostricshion of Bose-condensate and a system of neutral atoms. FNT, 2005, 31, No. 10, 1100-1102 (in Russian).

3. Lifshits E.M., Pitaevskii L.P. Statistical Physics, part 2. Nauka, Moscow, 1978, 620 p. (in Russian).

4. Rybalko A.S., Observation of electrical induction in a wave of the second sound in He II. FNT, 2004, 30, No. 11, 1321-1325 (in Russian); Low Temp. Phys, 2004, 30, No. 11, 994-998.

5. Brueckner K.A., Savada K., Bose-Einstein gas with repulsive interation: hard spheres at high density. Phys. Rev., 1957, 106, No. 6, 1128-1135.

6. Kosevich A.M., Electrical properties of a condensate of Bose-atoms. Ukr. Fiz. Zhurnal, 2005, 50, No. 8A, A130-A134 (in Russian).

\section{Спектр збуджень та електричні властивості конденсату Бозе-атомів}

\author{
А.М.Косевіч \\ Фізико-технічний інститут низьких температур ім.Б.Веркіна, \\ просп. Леніна, 47, Харків 61103 \\ Отримано 15 липня 2005 р., в остаточному вигляді - \\ 24 жовтня 2005 р.
}

Показано, що конденсат виродженого Бозе-газу нейтральних атомів володіє електричними властивостями, які відрізняються від тривіальної поляризації атомів у електричному полі. Вводиться поняття ізотропного квадрупольного моменту (IКМ) нейтрального атома. Розподіл IKМ відображає особливе просторове впорядкування в конденсаті і генерує розподіл електричного потенціалу. Розглядаються малі коливання Бозе-газу і отримується поправка до Боголюбівського спектру елементарних збуджень у виродженому Бозе-газі. Знайдено додатковий член у рівнянні Гроса-Пітаєвского, який відповідає за таку поправку та побудовано новий тип нелінійного рівняння Шредіннера. Оскільки Бозе-конденсат є споріднений 3 надплинною компонентою в Не II, то прояв його електричної поведінки може мати відношення до експериментально спостережуваної електричної поведінки надплинної рідини

Ключові слова: Бозе-конденсат, Боголюбівський спектр, електрична поляризація $\mathrm{He}$ II

PACS: 47.27.Eq, 67.40.Db 